\title{
ICE FLOW OVER BEDROCK PERTURBATIONS
}

\author{
W. F. BUDD \\ Antarctic Division, Department of Supply, 568 St Kilda Road, Melbourne, Victoria 3004, \\ Australia)
}

\begin{abstract}
The use of well known simple periodic solutions of the two-dimensional biharmonic stress equation for studying the flow over undulations of an ice mass of small surface slope is examined. The model considered is one in which most of the shear (deformation or sliding) takes place near the base and the upper part moves largely as a block, with longitudinal strain-rates varying linearly with the longitudinal stress deviations. For bedrock perturbations of a given wavelength the steady-state surface shape consists of similar waves but out of phase by $\frac{1}{2} \pi$, such that the steepest slope occurs over the highest bedrock; and the amplitude is reduced by a "damping factor", depending on the speed, viscosity, ice thickness and wavelength.

Minimum damping occurs for $\lambda_{\mathrm{m}} \approx 3.3$ times the ice thickness, while waves much longer or much shorter than this are almost completely damped out. The energy dissipation and the resistance to the ice flow is also a maximum for an undulation scale of several times the ice thickness, whereas the effects of small basal irregularities die out exponentially with distance into the ice, and only have an effect in so far as the average basal stress is related to the average surface slope. As a result of this a revision of present glacier sliding theories becomes possible.

Various predictions of the theory have been confirmed from spectral analysis of surface and bedrock profiles of ice caps.
\end{abstract}

RÉsumé. The French résumé will be found on p. 48 .

Zusammenfassung. Eisbewegung über Unebenheiten am Untergrund. Die Anwendbarkeit bekannter periodischer Lösungen der zweidimensionalen biharmonischen Spannungsgleichung auf das Studium der Bewegung einer Eismasse mit geringer Oberflächenneigung über Unebenheiten wird untersucht. In dem betrachteten Modell findet der Grossteil der Scherung (Verformung oder Gleiten) in der Nähe der Grundfläche statt, während der obere Teil sich weitgehend als Block bewegt. Die Deformationsgeschwindigkeit in Längsrichtung ändert sich linear mit den Abweichungen der Längsspannung. Bei Unebenheiten im Untergrund mit bekannter Wellenlänge zeigen sich auf der stationären Oberfläche ähnliche Wellen, jedoch mit einer Phasenverschiebung von $\frac{1}{2} \pi$, so dass die grössten Neigungen über den höchsten Stellen des Untergrundes auftreten. Die Amplitude wird durch einen "Dämpfungsfaktor" verringert, der von der Geschwindigkeit, der Viskosität, der Eisdicke und der Wellenlänge abhängt.

Die geringste Dämpfung tritt für $\lambda_{\mathrm{m}} \approx 3.3 \mathrm{mal}$ der Eisdicke ein, während Wellen mit sehr viel grösseren oder sehr viel kleineren Längen fast ganz verschwinden. Der Energieverlust und der Widerstand gegen die Eisbewegung ist ebenfalls bei einer Wellengrösse von mehrfacher Eisdicke maximal, während die Wirkung kleiner Unregelmässigkeiten am Untergrund exponentiell zum Abstand im Eis ausläuft und nur insoweit von Bedeutung ist, als die mittlere Spannung am Untergrund von der mittleren Oberflächenneigung abhängt. Als Folge hieraus scheint eine Überprüfung gegenwärtiger Theorien des Gletschergleitens möglich.

Verschiedene Voraussagen der Theorie wurden durch Geschwindigkeitsanalysen von Oberflächen- und Untergrundprofilen in Eisschilden bestätigt.

\section{Introduction}

\subsection{Background and previous work}

Undulations on the surface of ice caps with a predominant wavelength of the order of several times the ice thickness, have been described by many authors including Bourgoin (1956), Swithinbank (1959), Robin (1958, r967), Mälzer (1964), Robinson (1966), Budd ( 1966), Clough and others ( 1968), Beitzel (unpublished). Several authors have considered the influence of the bedrock shape on the surface profile (e.g. Bourgoin, r956; Nye, i959[b]; Lliboutry, I $964-65$, p. 635 ). Although certain qualitative features have been explained, Mock (1968) indicated that the simple relation between surface-slope variation and perturbation size was inadequate in explaining a measured profile. Robin (I967) made allowance for the effect of longitudinal stresses and strain-rates and showed that steady-state flow over bedrock variations resulted in surface slopes comparable with the measured slopes. The inclusion of the longitudinal stress changes in the analysis indicated that certain features of the bed profile were transmitted to the surface profile. However, in order to explain the existence of a predominant wavelength in the surface undulations it is necessary to include a further term from the equations of motion. 
In an earlier work the author (Budd, I969) considered ice flow over undulations using the integral of the equations of motion for the mean longitudinal stress-deviator gradient of a column, viz.

$$
\frac{\partial \bar{\sigma}_{x}^{\prime}}{\partial x}=\frac{1}{2} \rho g(\alpha-f)-\iint \frac{\partial^{2} \tau_{x z}}{\partial x^{2}} \mathrm{~d} z \mathrm{~d} z
$$

where $x$ is the longitudinal co-ordinate, $\alpha$ is the surface slope, $f=\tau_{\mathbf{b}} / \rho g$ is the basal friction where $\tau_{\mathrm{b}}$ is the base stress, $\rho$ the ice density and $g$ the gravitational acceleration, $\bar{\sigma}_{x}^{\prime}$ is the mean longitudinal stress deviator and $\tau_{x z}$ is the shear stress at point $(x, z)$.

With an additional assumption, based on measurements made at Wilkes, cf. Budd (rg68) and McLaren (I968), that the longitudinal strain-rate gradient is proportional to the surface slope deviations, Equation (I.I) was combined with the steady-state continuity equation to obtain the form of the ice surface slope,

$$
\alpha=\bar{\alpha}+\alpha_{1} \cos \omega x+\alpha_{2} \sin \omega x
$$

for steady-state flow over bedrock undulations of slope,

$$
\beta=\beta_{1} \cos \omega x
$$

where $\lambda=\omega / 2 \pi$ is the wavelength of the undulations.

The results of that analysis, viz.

where

$$
\begin{gathered}
\alpha_{\mathrm{I}}=\frac{\beta_{\mathrm{I}}}{\mathrm{I}+\psi^{2}} \quad \text { and } \quad \alpha_{2}=\frac{\psi \beta_{\mathrm{I}}}{\mathrm{I}+\psi^{2}} \\
\psi=\frac{\rho g Z^{2}}{2 B V}\left(\mathrm{I}+\frac{4 \pi^{2} Z^{2}}{3^{\lambda^{2}}}\right) \\
=\frac{\rho g Z^{2}}{2 B V}\left(\frac{\mathrm{I}}{\omega Z}+\frac{\omega Z}{3}\right)
\end{gathered}
$$

where $Z$ is the ice thickness, $V$ is the average horizontal velocity and $B$ is the ice flow-law parameter indicate that since $\psi$ is large, the surface slope is out of phase with the bedrock by approximately $\frac{1}{2} \pi$, such that the maximum surface slope occurs over the maximum bedrock elevation. This qualitative result has been demonstrated for waves with wavelength about $30 \mathrm{~km}$ by Bourgoin (I956) and was also inferred by Nye (I959[a]) from the simple velocity basal stress relationship.

Equations (I.4) and (I.5) also indicate that the damping factor $\psi \approx \alpha_{2} / \beta_{1}$ increases with the square of the ice thickness and is inversely proportional to the velocity and the viscosity flow parameter $B$. Furthermore the damping factor has a frequency dependence with a minimum for $\lambda / Z=2 \pi / \sqrt{ } 3 \approx 3.6$, i.e. for wavelengths between 3 and 4 times the ice thickness.

This result implies that both the very long and very short waves are predominantly damped out so that given for example a uniform distribution of bedrock elevations one may expect the surface undulations to have a predominant wavelength about $\lambda=3.6 Z$.

Equations (I.4) and (I.5) are useful for determining how the flow parameter varies over an ice mass from measurements of the surface and bedrock profiles together with the velocity $V$.

Some of the predictions of this earlier theory have already been confirmed from the analysis of surface and bedrock profiles by Beitzel (unpublished), D. Carter* and W. F. Budd and D. Carter. $\dagger$

The major difficulty in this construction was that a somewhat arbitrary assumption had to be made on how the term $\partial^{2} \tau_{x z} / \partial x^{2}$ of Equation (I.I) varies with depth. This term, which was neglected by Robin ( 1967$)$, is responsible for the short waves being damped out. The following analysis is much more general and attacks the problem by means of stress functions using an approach similar to that of Yosida (1964). Report.]

* Wilkes ice cap project 1967 , investigations by radar sounding. [To be published as an ANARE Scientific

$\dagger$ Analysis of the relation between the surface and bed-rock of ice caps. [In preparation.] 


\section{I.2. The model to be treated}

Yosida ( 1964 ) considered Newtonian viscous flow with no slip of a medium of uniform thickness down a uniform slope with small harmonic undulations superimposed on it. He obtained a general solution by superimposing the simple solution of uniform flow without the undulations upon harmonic solutions of the biharmonic stress equation.

The model to be treated here is somewhat more general than that considered by Yosida. The aim is to allow extension to non-Newtonian flow but because the flow parameters in general depend on the temperature and stress distributions throughout the ice mass the discussion here is restricted to a simplified model. It can readily be extended to a more general model when the temperature, stress, or flow-parameter distribution throughout the ice mass is known.

Analysis of the Wilkes ice cap data (Budd, 1968; McLaren, 1968) indicates that for small longitudinal stress deviations the longitudinal strain-rates varied almost proportionally. This suggests that for these low stresses the effective flow law is not far from linear. On the other hand most of the vertical shear occurs in the basal layers such that variation in velocity is of a high power of stress.

Hence we consider the model of a uniform mass of ice sliding down a slope, rather than using the no-slip condition, with a power-law distribution of the horizontal velocity in the vertical and a constant viscosity reaction of longitudinal strain-rate to small perturbations in longitudinal stresses caused by undulations of the bed. An extension of the analysis allows the "viscosity" to be prescribed as a function of depth (or stress and temperature). This model represents a closer approximation to the case of typical ice masses moving down a slope with most of the shear taking place in the lower layers, and it is not of great consequence whether the motion consists of direct sliding between a sharp ice-rock interface or a combination of sliding and high shear in various transition zones of moraine and ice mixture near the base.

The resistance to the ice motion may be considered as made up of two parts. First there is the uniform basal stress and horizontal shear for flow down a flat plane. This basal stress is equivalent to a friction coefficient $f,\left(f=\tau_{\mathrm{b}} / \rho g Z\right)$ which from the integration of Equation (I.I) along the ice mass can be seen to be approximately equal to the average surface slope. Secondly due to the irregularities in the bed additional stresses and strain-rates $\left(\sigma_{i j}\right.$ and $\left.\dot{\epsilon}_{i j}\right)$ are set up in the ice.

The energy dissipation $\iint \sigma_{i j} \dot{\epsilon}_{i j} \mathrm{~d} z \mathrm{~d} x$ over all such irregularities throughout the ice mass, corresponds to a net reduction in the rate of work done by gravity in moving the ice down the slope.

The aim here is to calculate the effect of such irregularities. Although a general solution of this problem for a non-Newtonian medium analogous to that of Yosida for a viscous medium is complex it will be shown in this paper that, for the model described above, elementary solutions of the biharmonic equation for the stress function exist which satisfy the steadystate continuity equation and the boundary stress and velocity conditions to a close approximation for an ice mass of small surface slope.

\section{Equations of motion, the stress function and the biharmonic Equation}

The use of the stress function $\phi$ and the biharmonic equation $\nabla^{4} \phi=0$ in two dimensions has been well established for obtaining solutions for plane strain in elastic media (cf. Timoshenko, 1934; Sokolnikoff and Specht, 1956). Corresponding results apply for solutions of stresses and velocities in Newtonian viscous media using stream functions (cf. Lamb, I932; Jaeger, I962). The application of some of these results to ice flow have been discussed by Shumskiy (1967).

Although some of this standard work is repeated, the equations are derived here in order to indicate which of the basic results apply to non-linear flow and at what stage it is necessary to invoke the condition of constant viscosity. 
2.I. Steady flow down a uniform slope

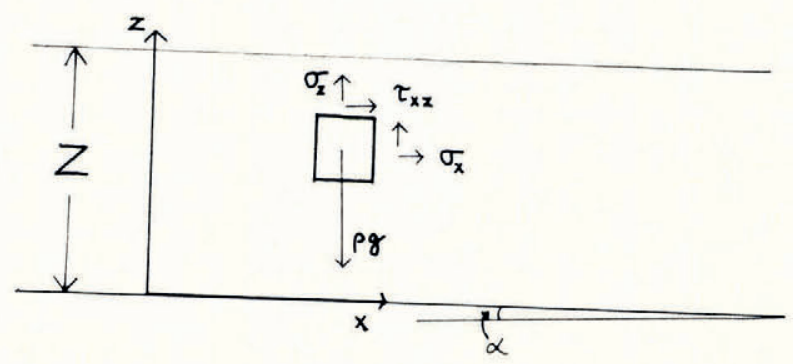

Fig. I. Uniform flow down a constant slope $\alpha$.

Consider a two-dimensional section of an ice mass of uniform density $\rho$ and uniform thickness $Z$ on an inclined plane of slope $\alpha$. The velocity solution for this simple model was given by Nye (1952). Nevertheless a derivation is given here to emphasize the conditions upon which the velocity and stress solutions depend. Take the $x$-axis down the slope and $z$ perpendicular upwards. Denote the stress components with respect to these axes by $\sigma_{i j}$ or $\left(\sigma_{x}, \tau_{x z}, \sigma_{z}\right)$.

The equations of motion for slow steady flow (inertial components neglected) may be written.

$$
\begin{aligned}
& \frac{\partial \sigma_{x}}{\partial x}+\frac{\partial \tau_{x z}}{\partial z}+\rho g \sin \alpha=0 \\
& \frac{\partial \sigma_{z}}{\partial z}+\frac{\partial \tau_{x z}}{\partial x}-\rho g \cos \alpha=0 .
\end{aligned}
$$

Let $u, v$ be the velocity components in the $x$ and $z$ directions at the point $(x, z)$. The components of strain-rate $\left(\dot{\epsilon}_{i j}\right)$ are then given by,

$$
\dot{\epsilon}_{x}=\frac{\partial u}{\partial x}, \quad \dot{\epsilon}_{z}=\frac{\partial v}{\partial z}, \quad \dot{\gamma}_{x z}=\frac{\mathrm{I}}{2}\left(\frac{\partial u}{\partial z}+\frac{\partial v}{\partial x}\right) .
$$

For this simple model the compatability conditions are not required. Following Yosida we consider solutions of the equations of motion first for zero longitudinal variation and then for harmonic perturbations from this state.

The complete solution is then obtained from the principle of superposition.

It is necessary to point out here that whereas the principle of superposition applies for linear deformation the generalization to non-linear flow presents further difficulties. However, here we consider only perturbations of stresses and regard the combined velocity distribution as calculated from a flow parameter which is a function of the combined stresses.

For zero longitudinal variation $\frac{\partial \sigma_{x}}{\partial x}=\frac{\partial \tau_{x z}}{\partial x}=0$ and Equations (2.I) and (2.2) integrate to

$$
\sigma_{x}=\sigma_{z}^{0}=-\rho g \cos \alpha(Z-z), \quad \tau_{x z}^{0}=\rho g \sin \alpha(Z-z)
$$

where the boundary conditions at the surface have been taken as normal and shear surface stresses are zero,

$$
\left.\left.\sigma_{\mathrm{n}}\right)_{\mathrm{s}}=\tau_{\mathrm{nt}}\right)_{\mathrm{s}}=\mathrm{o}
$$

(the atmospheric pressure is for the moment neglected).

We note here that the stress solution is independent of the flow law and the velocity solution, depending only on the equations of motion, the uniformity in the $x$-direction and 
the boundary stresses. Now, if a flow law is given, the velocity profile may be determined, thus if

$$
\dot{\epsilon}_{i j}=\frac{\sigma_{i j}^{\prime}}{\eta}
$$

where $\sigma_{i j}^{\prime}$ is the stress deviator,

$$
\sigma_{x}^{\prime}=\frac{1}{2}\left(\sigma_{x}-\sigma_{z}\right), \quad \sigma_{z}^{\prime}=\frac{1}{2}\left(\sigma_{z}-\sigma_{x}\right), \quad \tau_{x z}^{\prime}=\tau_{x z}
$$

and $\eta$ is a function of the octahedral shear stress

and the temperature of the ice.

$$
\tau_{0}=\frac{1}{3}\left(\sigma_{i j}^{\prime} \sigma_{i j}^{\prime}\right)^{1 / 2}
$$

For the particular case of a power law for flow we may write for Equation (2.6)

$$
\dot{\epsilon}_{i j}=\frac{\tau^{n-1}}{B^{n}} \sigma_{i j}^{\prime}
$$

where $B$ is dependent on temperature, but constant with stress.

Using this in the third of the stress solutions (2.4) together with Equation (2.3) gives,

where $\zeta=(z-z)$.

$$
\frac{\mathrm{d} u}{\mathrm{~d} \zeta}=2(\rho g \sin \alpha \zeta / B)^{n}
$$

This equation integrates to,

$$
u_{\zeta}=u_{\mathrm{b}}+\frac{2}{n+\mathrm{I}}\left(\frac{\rho g \sin \alpha}{B}\right)^{n}\left(Z^{n+\mathbf{I}}-\zeta^{n+\mathrm{I}}\right)
$$

where $u_{\mathrm{b}}$ is an unspecified velocity of sliding at the base, $z=0$.

\subsection{Harmonic perturbations from uniform flow}

Next we consider perturbations from the uniform solution. Consider the equations of motion for zero external forces,

$$
\begin{aligned}
& \frac{\partial \sigma_{x}}{\partial x}+\frac{\partial \tau_{x z}}{\partial z}=0, \\
& \frac{\partial \sigma_{z}}{\partial z}+\frac{\partial \tau_{x z}}{\partial x}=0 .
\end{aligned}
$$

The complete solution to the problem of flow over undulations can be obtained by superimposing solutions of these on the solutions (2.4) such that all the boundary conditions are satisfied.

To obtain solutions of Equations (2.12) and (2.13) we make use of the stress function.

For any function $\phi$ the equations of motion (2.12) and (2.13) are satisfied identically by taking stress components as,

$$
\sigma_{x}=\frac{\partial^{2} \phi}{\partial z^{2}}, \quad \sigma_{z}=\frac{\partial^{2} \phi}{\partial x^{2}}, \quad \tau_{x z}=-\frac{\partial^{2} \phi}{\partial x \partial z} .
$$

The function $\phi$ must also be chosen such that the boundary conditions of stress are satisfied.

Since Equations (2.12) and (2.13) constitute two equations in three unknowns, in general this is not sufficient to provide a solution for the stresses. Hence it is often necessary to introduce a flow law (stress-strain-rate relation) and together with the equation of compatibility for the strain-rates a solution may then be obtained.

The equations of compatibility are obtained by differentiations of Equations (2.3) as,

$$
\frac{\hat{\partial}^{2} \dot{\epsilon}_{x}}{\partial z^{2}}+\frac{\partial^{2} \dot{\epsilon}_{z}}{\partial x^{2}}=2 \frac{\hat{\partial}^{2} \dot{\gamma} x z}{\partial x \partial z}
$$


The flow law may be written,

$$
\frac{\frac{1}{2}\left(\sigma_{x}-\sigma_{z}\right)}{\partial u / \partial x}=\frac{\frac{1}{2}\left(\sigma_{z}-\sigma_{x}\right)}{\partial v / \partial z}=\frac{\tau_{x z}}{\frac{1}{2}(\partial u / \partial z+\partial v / \partial x)}=\frac{\tau}{\dot{\epsilon}}=2 \eta
$$

where $\eta$ is the coefficient of viscosity, which for Newtonian flow is constant throughout the medium. Equations (2.16) also hold for non-Newtonian and plastic flow; however, the parameter $\eta$ is then not an absolute constant, but varies with position in the medium depending on the stress and temperature according to the flow law or yield criterion.

First let us examine the case of Newtonian flow (constant $\eta$ ). Direct substitution of the stresses from Equations (2.16) in the compatability conditions (2.15) together with the stressfunction equations (2.14) then yields the biharmonic equation for $\phi$,

$$
\nabla^{4} \phi=\frac{\partial^{4} \phi}{\partial x^{4}}+4 \frac{\partial^{4} \phi}{\partial x^{2} \partial z^{2}}+\frac{\partial^{4} \phi}{\partial z^{4}}=0 .
$$

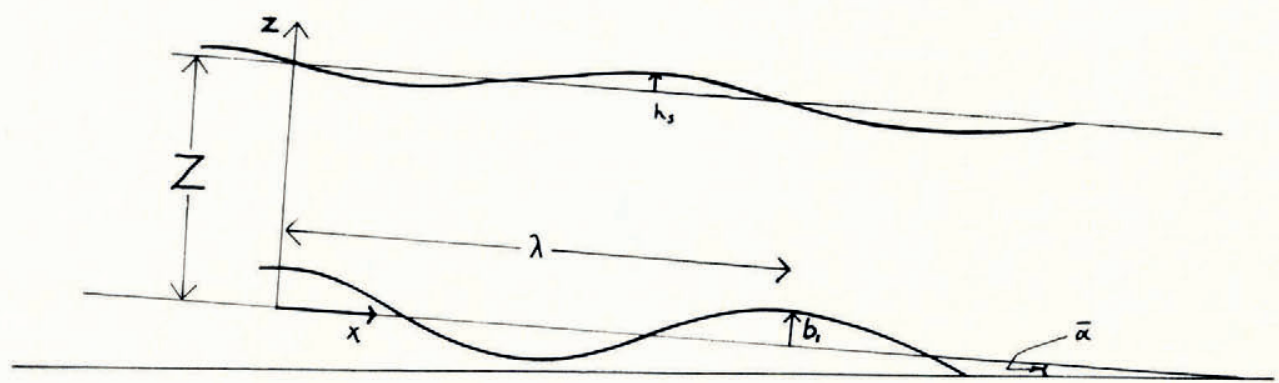

Fig. 2. Flow down a slope with harmonic perturbations.

Now if the bed of our uniform model (Fig. I) has undulations of wavelength $\lambda=\omega / 2 \pi$ superimposed on the uniform slope $\bar{\alpha}$ (Fig. 2), we may write for the bed,

$$
b=b_{1} \cos \omega x \quad \text { where } b_{\mathrm{I}} \ll z \text {. }
$$

We suppose that the surface may be given by

$$
z+h=Z+h_{1} \cos \omega x+h_{2} \sin \omega x \text {. }
$$

Hence, for a viscous medium, we consider solutions of the biharmonic equation which are periodic in $x$ by taking,

where $f_{1}$ and $f_{2}$ are functions of $z$ only.

$$
\phi=f_{1}(z) \cos \omega x+f_{2}(z) \sin \omega x
$$

Substituting $\phi$ in Equation (2.17) yields the following ordinary differential equation for the $f_{\mathrm{s}}$ in $z$ (the primes denote differentiation with respect to $z$ ),

The general integral of this equation is,

$$
\omega^{4}-2 \omega^{2} f^{\prime \prime}+f^{\prime \prime \prime \prime}=0 .
$$

$$
f=\left(a_{\mathrm{r}}+a_{2} z\right) \mathrm{e}^{\omega z}+\left(a_{3}+a_{4} z\right) \mathrm{e}^{-\omega z}
$$

where the $a_{\mathrm{s}}$ are arbitrary constants.

Therefore an appropriate general simple periodic solution of the biharmonic equation may be obtained as,

$\phi=\left\{\left(a_{1}+a_{2} z\right) \mathrm{e}^{\omega z}+\left(a_{3}+a_{4} z\right) \mathrm{e}^{-\omega z}\right\} \cos \omega x+\left\{\left(a_{5}+a_{6} z\right) \mathrm{e}^{\omega z}+\left(a_{7}+a_{8} z\right) \mathrm{e}^{-\omega z}\right\} \sin \omega x$.

It is worth noting here that since solutions for different frequencies $\omega$ are additive a general solution of the form,

$$
\phi=\sum_{n=1}^{\infty}\left(f_{\mathrm{r}, n} \cos n \omega x+f_{2, n} \sin n \omega x\right)
$$


may be used in conjunction with Fourier series or integrals to fit any more general form of bed shape.

Solutions of the form of Equation (2.22) were used by Yosida (1964) in his study of viscous flow with no slip over undulations down a uniform slope. We now consider the extension of this analysis to non-linear flow. This, at first sight is impeded by the equation of compatibility which for stresses then becomes

where $\eta$ is not constant.

$$
\frac{\partial^{2}\left(\sigma_{x}-\sigma_{z}\right) / \eta}{\partial x^{2}}+\frac{\partial^{2}\left(\sigma_{z}-\sigma_{x}\right) / \eta}{\partial z^{2}}=4 \frac{\partial^{2}\left(\tau_{x z} / \eta\right)}{\partial x \partial z}
$$

For this study however, we are considering perturbations from the stress solution (2.4). This means we can consider the equations of motion $(2.12,2.13)$ being in terms of stress deviators.

The continuity relation for an incompressible medium,

$$
\dot{\epsilon}_{x}=-\dot{\epsilon}_{z},
$$

together with the generalized flow law (2.16) then implies

$$
\sigma_{x}=-\sigma_{z} .
$$

In terms of the stress function we then obtain Laplace's equation,

$$
\frac{\partial^{2} \phi}{\partial z^{2}}+\frac{\partial^{2} \phi}{\partial x^{2}}=\nabla^{2} \phi=0
$$

which is a special case of the more general biharmonic equation. The corresponding solution for periodic variations in $x$ reduce to,

$$
\phi=\left(A_{\mathrm{I}} \mathrm{e}^{\omega z}+A_{2} \mathrm{e}^{-\omega z}\right) \cos \omega x+\left(A_{3} \mathrm{e}^{\omega z}+A_{4} \mathrm{e}^{-\omega z}\right) \sin \omega x
$$

which is equivalent to dropping the terms in $z$ from Equation (2.22).

At this stage it has not been necessary to invoke the flow law, merely the condition that for a given stress the strain-rate components are proportional to the stress deviator components (which applies for isotropic flow laws from constant viscosity to plastic).

\section{The BOUNDARY CONDITIONS}

The problem of fitting the harmonic solution (2.28) exactly to boundary values of stress and velocity can be very difficult. Here we examine the boundary conditions and find that certain simplifications can be made which are appropriate for small surface slopes as found on large ice masses.

If $\sigma_{\mathrm{n}}$ and $\tau_{\mathrm{nt}}$ denote the normal stress and traction at the surface then the condition that the surface traction is zero, and the normal pressure is the atmospheric pressure $p$, may be written,

$$
\left.\left.\sigma_{\mathrm{n}}\right)_{\mathrm{s}}=p \quad \text { and } \quad \tau_{\mathrm{nt}}\right)_{\mathrm{s}}=\mathrm{o}
$$

where the s subscript indicates that the value is taken along the curved surface contour.

The second of Equations (3.1) implies,

where $\alpha$ is the surface slope.

$$
\frac{2 \tau_{x z}}{\left(\sigma_{x}-\sigma_{y}\right)}=2 \tan \alpha
$$

This equation is difficult to satisfy exactly, but for small slopes the right-hand side simply becomes $2 \alpha$. However, zeros in $\left(\sigma_{x}-\sigma_{y}\right)$ still cause problems here. To avoid these we make use of the well known method of solutions for a surface with harmonic loading (cf. Jaeger, I964; Lamb, I932) and suppose that for small surface slopes the first of Equations (3.I) can be replaced by,

$$
\begin{aligned}
\left.\sigma_{z}\right)_{Z} & =\rho g h \\
& =\rho g\left(h_{1} \cos \omega x+h_{2} \sin \omega x\right)
\end{aligned}
$$


where the subscript $Z$ denotes the value at $z=Z$, and $h_{1}+h_{2} \ll Z$. It will be found that both $\left.\tau_{x z}\right)_{Z}$ and $\left.\tau_{\mathrm{nt}}\right)_{\mathrm{s}}$ are small (cf. section 4.2) and hence this simplification involves no serious loss of generality.

The velocity boundary conditions are determined by the shape of the base contour, the mean down-slope velocity and the equation of continuity.

The general equation of continuity for a steady-state ice mass of thickness $Z$ moving with a mean velocity $V$, and where the accumulation rate at the surface is $A$ may be written,

or

$$
\begin{gathered}
\frac{\mathrm{d}(V Z)}{\mathrm{d} x}=A \\
V \frac{\mathrm{d} Z}{\mathrm{~d} x}+Z \frac{\mathrm{d} V}{\mathrm{~d} x}=A .
\end{gathered}
$$

Now if we are considering just harmonic perturbations in $V$ and $Z$ from regional averages we may write,

and

$$
\frac{\mathrm{d} Z}{\mathrm{~d} x}=\bar{\beta}-\bar{\alpha}+\left(\beta_{1}-\alpha_{1}\right) \cos \omega x-\alpha_{2} \sin \omega x
$$

$$
\frac{\mathrm{d} V}{\mathrm{~d} x}=\bar{\epsilon}_{\mathrm{o}}+\bar{\epsilon}_{1} \cos \omega x+\bar{\epsilon}_{2} \sin \omega x \text {. }
$$

Substituting Equations (3.6) and (3.7) in Equation (3.5) and equating individual terms in the sine and cosine since they apply for all $x$, yields,

$$
\begin{aligned}
V(\bar{\beta}-\bar{\alpha})+H \bar{\epsilon}_{0} & =A \\
\beta_{1}-\alpha_{1} & =-z \bar{\epsilon}_{1} / V \\
\alpha_{2} & =z \bar{\epsilon}_{2} / V
\end{aligned}
$$

Equation (3.8) simply expresses the mean continuity condition for the regionally smoothed values independent of the undulations. Here we need only consider the effects associated with the perturbations. The modifications necessary in the case of associated variations in accumulation rate have been given by Budd ( 1969 , section 5.4.1).

Since

$$
\bar{\epsilon}_{\mathrm{I}}=\frac{\mathrm{I}}{z} \int_{0}^{z} \frac{\partial u}{\partial x} \mathrm{~d} z=-\frac{\mathrm{I}}{Z} \int_{0}^{z} \frac{\partial v}{\partial z} \mathrm{~d} z
$$

and

$$
\beta=\frac{\mathrm{d} b}{\mathrm{~d} x}
$$

where $b$ is the bedrock elevation (above $z=0$ ), Equations (3.9) and (3.10) may be alternatively written,

and

$$
\begin{aligned}
& V \frac{\mathrm{d} b}{\mathrm{~d} x}=V \beta=v_{\mathrm{b}} \\
& V \frac{\mathrm{d} h}{\mathrm{~d} x}=V \alpha=v_{Z}
\end{aligned}
$$

Hence we now look for solutions of the form,

$$
\phi=\left(A_{1} \mathrm{e}^{\omega z}+A_{2} \mathrm{e}^{-\omega z}\right) \cos \omega x+\left(A_{3} \mathrm{e}^{\omega z}+A_{4} \mathrm{e}^{-\omega z}\right) \sin \omega x
$$

subject to the boundary conditions $(3 \cdot 3),(3.13),(3.14)$. These are just sufficient to determine the four coefficients $A_{i}$, and the shape of the steady-state surface $\alpha$. 
4. Determination of stress-function coefficients AND the form of the steady-STAte SURFACE

4. I. Satisfying the boundary conditions

For a stress function of the form

$$
\phi=\left(A_{1} \mathrm{e}^{-\omega z}+A_{2} \mathrm{e}^{\omega z}\right) \sin \omega x+\left(A_{3} \mathrm{e}^{-\omega z}+A_{4} \mathrm{e}^{(\omega z}\right) \cos \omega x
$$

the stresses from Equations (2.14) are given by,

$$
\begin{aligned}
\sigma_{x} & =\omega^{2}\left(A_{1} \mathrm{e}^{-\omega z}+A_{2} \mathrm{e}^{\omega z}\right) \sin \omega x+\omega^{2}\left(A_{3} \mathrm{e}^{-\omega z}+A_{4} \mathrm{e}^{\omega z}\right) \cos \omega x, \\
\sigma_{z} & =-\omega^{2}\left(A_{1} \mathrm{e}^{-\omega z}+A_{2} \mathrm{e}^{\omega z}\right) \sin \omega x-\omega^{2}\left(A_{3} \mathrm{e}^{-\omega z}+A_{4} \mathrm{e}^{\omega z}\right) \cos \omega x, \\
\tau_{x z} & =\omega^{2}\left(-A_{1} \mathrm{e}^{-\omega z}+A_{2} \mathrm{e}^{\omega z}\right) \cos \omega x-\omega^{2}\left(-A_{3} \mathrm{e}^{-\omega z}+A_{4} \mathrm{e}^{\omega z}\right) \sin \omega x, \\
\frac{1}{2}\left(\sigma_{x}-\sigma_{z}\right) & =\omega^{2}\left(A_{1} \mathrm{e}^{-\omega z}+A_{2} \mathrm{e}^{\omega z}\right) \sin \omega x+\omega^{2}\left(A_{3} \mathrm{e}^{-\omega z}+A_{4} \mathrm{e}^{\omega z}\right) \cos \omega x .
\end{aligned}
$$

From Equations (2.16)

$$
2 \eta \frac{\partial u}{\partial x}=-2 \eta \frac{\partial v}{\partial z}=\frac{1}{2}\left(\sigma_{x}-\sigma_{z}\right) .
$$

At this stage, for the moment, we consider the medium to react to longitudinal stress variations with constant viscosity. If $\eta$ were prescribed as a function of depth $z$, then this could be incorporated for more general flow laws in the following integrations.

Integrating $\frac{1}{2}\left(\sigma_{x}-\sigma_{z}\right)$ then gives, with the boundaries unspecified,

$$
\begin{aligned}
& 2 \eta u=-\omega\left(A_{1} \mathrm{e}^{-\omega z}+A_{2} \mathrm{e}^{\omega z}\right) \cos \omega x+\omega\left(A_{3} \mathrm{e}^{-\omega z}+A_{4} \mathrm{e}^{\omega z}\right) \sin \omega x, \\
& 2 \eta v=-\omega\left(-A_{1} \mathrm{e}^{-\omega z}+A_{2} \mathrm{e}^{\omega z}\right) \sin \omega x-\omega\left(-A_{3} \mathrm{e}^{-\omega z}+A_{4} \mathrm{e}^{\omega z}\right) \cos \omega x .
\end{aligned}
$$

From the boundary condition (3.13) $v_{\mathrm{b}}=V \beta$ at the base $z=0$. Hence

$$
\begin{aligned}
2 \eta v_{\mathrm{b}} & =-\omega\left(-A_{1}+A_{2}\right) \sin \omega x-\omega\left(-A_{3}+A_{4}\right) \cos \omega x \\
& =2 \eta V \beta_{1} \sin \omega x .
\end{aligned}
$$

Hence

$$
\left.\begin{array}{rlrlrl}
\beta_{\mathrm{I}} & =-\frac{\omega\left(A_{2}-A_{1}\right)}{2 \eta V} & \text { or } & & \left(A_{2}-A_{1}\right) & =-\frac{2 \eta V \beta_{\mathrm{I}}}{\omega} \\
\mathrm{o} & =-A_{3}+A_{4} & \text { or } & A_{3} & =A_{4}
\end{array}\right\}
$$

and

For the upper surface, $z=Z, v_{Z}=V \alpha=V \frac{\mathrm{d} h}{\mathrm{~d} x}$.

Hence

$$
\begin{aligned}
\alpha & =\frac{-\omega}{2 \eta V}\left\{\left(-A_{1} \mathrm{e}^{-\omega Z}+A_{2} \mathrm{e}^{\omega Z}\right) \sin \omega x+A_{3}\left(\mathrm{e}^{\omega Z}-\mathrm{e}^{-\omega Z}\right) \cos \omega x\right\} \\
& =\alpha_{1} \sin \omega x+\alpha_{2} \cos \omega x
\end{aligned}
$$

where

$$
\alpha_{1}=\frac{-\omega}{2 \eta V}\left(-A_{1} \mathrm{e}^{-\omega Z}+A_{2} \mathrm{e}^{\omega Z}\right) \quad \text { and } \quad \alpha_{2}=\frac{-\omega}{2 \eta V} A_{3}\left(\mathrm{e}^{\omega Z Z}-\mathrm{e}^{-\omega Z}\right) \text {. }
$$

Hence

$$
h=\frac{\mathrm{I}}{2 \eta V}\left\{\left(-A_{1} \mathrm{e}^{-\omega Z}+A_{2} \mathrm{e}^{\omega Z}\right) \cos \omega x-A_{3}\left(\mathrm{e}^{\omega Z}-\mathrm{e}^{-\omega Z}\right) \sin \omega x\right\} .
$$

From the boundary condition Equation (3.3),

$$
\begin{aligned}
\left.\sigma_{z}\right)_{\mathrm{s}} & =\rho g h \\
& =\frac{\rho g}{2 \eta V}\left\{\left(-A_{1} \mathrm{e}^{-\omega Z}+A_{2} \mathrm{e}^{\omega Z}\right) \cos \omega x-A_{3}\left(\mathrm{e}^{\omega Z Z}-\mathrm{e}^{-\omega Z}\right) \sin \omega x\right\} .
\end{aligned}
$$

Also from Equation (4.3) above for $z=Z$,

$$
\left.\sigma_{z}\right)_{\mathrm{s}}=-\omega^{2}\left(+A_{1} \mathrm{e}^{-\omega Z}+A_{2} \mathrm{e}^{\omega Z}\right) \sin \omega x-\omega^{2} A_{3}\left(\mathrm{e}^{\omega Z}+\mathrm{e}^{-\omega Z}\right) \cos \omega x .
$$

These equations hold for all $x$ so the $A$ may be found by equating coefficients of $\sin x$, and $\cos x$ in Equations (4.15) and (4.16),

$$
-A_{3} c=-\chi A_{1} \mathrm{e}^{-\omega Z}+\chi A_{2} \mathrm{e}^{\omega Z}
$$


where we write

$$
\chi A_{3} s=A_{\mathrm{I}} \mathrm{e}^{-\omega Z}+A_{2} \mathrm{e}^{\omega Z},
$$

and

$$
c=2 \cosh \omega Z, \quad s=2 \sinh \omega Z
$$

We can solve Equations (4.17) and (4.18) for $A_{3}, A_{2}$ in terms of $A_{1}$ by adding then subtracting as follows:

Hence

$$
\begin{aligned}
A_{3}\left(\chi^{2} s-c\right) & =2 \chi A_{2} \mathrm{e}^{\omega} Z \\
-A_{3}\left(c+\chi^{2} s\right) & =-2 \chi A_{1} \mathrm{e}^{-\omega Z} .
\end{aligned}
$$

and

$$
A_{3}=\frac{2 \chi A_{1} \mathrm{e}^{-\omega Z}}{c+\chi^{2} s}
$$

$$
A_{2}=\frac{A_{3}\left(\chi^{2} s-c\right)}{2 \chi \mathrm{e}^{\omega Z}}
$$

which, from Equation (4.23)

$$
=A_{1} \mathrm{e}^{-2 \omega Z}\left(\frac{\chi^{2} s-c}{\chi^{2} s+c}\right) .
$$

\subsection{Relation between surface and bedrock}

The surface slope components $\alpha_{1}, \alpha_{2}$ can now be determined in terms of the basal slope $\beta_{1}$ since from Equations (4.9) and (4.12),

$$
\frac{\alpha_{1}}{\beta_{\mathrm{I}}}=\frac{A_{2} \mathrm{e}^{\omega Z}-A_{\mathrm{I}} \mathrm{e}^{-\omega Z}}{A_{2}-A_{\mathrm{I}}}, \quad \frac{\alpha_{2}}{\beta_{\mathrm{I}}}=\frac{A_{3} \cdot s}{A_{2}-A_{\mathrm{I}}} .
$$

Now from Equation (4.25),

and

$$
\begin{aligned}
& A_{2} \mathrm{e}^{\omega Z}-A_{\mathrm{I}} \mathrm{e}^{-\omega Z}=-A_{\mathrm{I}} \mathrm{e}^{-\omega Z}\left(\mathrm{I}-\frac{\chi^{2} s-c}{\chi^{2} s+c}\right) \\
& =-\frac{2 A_{\mathrm{I}} c \mathrm{e}^{-\omega} Z}{\chi^{2} s+c}, \\
& A_{2}-A_{\mathrm{I}}=-A_{\mathrm{I}}\left\{\mathrm{I}-\mathrm{e}^{-2 \omega Z}\left(\frac{\chi^{2} s-c}{\chi^{2} s+c}\right)\right\} \\
& =-A_{\mathrm{r}}\left\{\frac{\chi^{2} s\left(\mathrm{I}-\mathrm{e}^{-2 \omega Z}\right)+c\left(\mathrm{I}+\mathrm{e}^{-2 \omega Z}\right)}{\chi^{2} s+c}\right\} .
\end{aligned}
$$

Hence from Equations (4.26), (4.23) and (4.27),

$$
\frac{\alpha_{2}}{\alpha_{\mathrm{I}}}=-\frac{A_{3} \cdot s}{A_{2} \mathrm{e}^{\omega Z}-A_{1} \mathrm{e}^{-\omega Z}}=\chi s,
$$

and from Equations (4.26), (4.23) and (4.28),

$$
\begin{aligned}
\frac{\alpha_{2}}{\beta_{1}}=-\frac{A_{3} \cdot s}{A_{2}-A_{1}} & =\frac{2 \chi s \mathrm{e}^{-\omega Z}}{\chi^{2} s\left(\mathrm{I}-\mathrm{e}^{-2 \omega Z}\right)+c\left(\mathrm{I}+\mathrm{e}^{-2 \omega Z}\right)} \\
& =\frac{2 \chi s}{\chi^{2} s^{2}+c^{2}} .
\end{aligned}
$$

Similarly

$$
\begin{aligned}
\frac{\alpha_{\mathrm{I}}}{\beta_{\mathrm{I}}}=\frac{A_{2} \mathrm{e}^{\omega Z}-A_{\mathrm{I}} \mathrm{e}^{-\omega Z}}{A_{2}-A_{\mathrm{I}}} & =\frac{2 c \mathrm{e}^{-\omega Z}}{\chi^{2} s\left(\mathrm{I}-\mathrm{e}^{-2 \omega Z}\right)+c\left(\mathrm{I}+\mathrm{e}^{-2 \omega Z}\right)} \\
& =\frac{2 c}{\chi^{2} s^{2}+c^{2}} .
\end{aligned}
$$

The amplitude of the surface undulations is given by $\alpha_{\mathrm{S}}=\left(\alpha_{1}^{2}+\alpha_{2}^{2}\right)^{1 / 2}$. 
We define the damping factor $\psi$ as the ratio of basal to surface amplitudes. Hence from Equations (4.30), (4.3I),

$$
\psi=\frac{\beta_{1}}{\alpha_{\mathrm{B}}}=-\frac{\chi^{2} s^{2}+c^{2}}{2\left(\chi^{2} s^{2}+c^{2}\right)^{1 / 2}}=-\frac{1}{2}\left(\chi^{2} s^{2}+c^{2}\right)^{\frac{1}{2}} .
$$

Since, as will be shown shortly, $\chi^{s}$ is generally large,

which from Equation (4.20), gives

$$
\psi \approx \frac{1}{2} \chi^{s}
$$

$$
\begin{aligned}
\psi & =\frac{\rho g Z^{2}\left(\mathrm{e}^{\omega Z}-\mathrm{e}^{-\omega Z}\right)}{4 \eta V(\omega Z)^{2}} \\
& =\frac{\rho g Z^{2}}{2 \eta V}\left\{\frac{\mathrm{I}}{\omega Z}+\frac{\omega Z}{3 !}+\frac{(\omega Z)^{3}}{5 !}+\ldots\right\} .
\end{aligned}
$$

This shows that the earlier damping factor of Budd (I969) (Equation (I.6)) may be regarded as a good first approximation, except that $B=2 \eta$ is the viscosity parameter. The factor of 2 difference shows an error in a basic assumption of the earlier theory and the import of this will be discussed in detail in a separate paper. Otherwise most of the predictions of Budd (1969) carry over.

In order to gauge the order of magnitudes involved we note that for viscous flow with zero sliding the surface velocity is given by,

$$
V_{\mathrm{s}}=\frac{\rho g \bar{\alpha} \bar{Z}^{2}}{4 \eta} .
$$

Hence if we replace $\rho g Z^{2} / 4 \eta V$ by $\bar{\alpha}$, the mean regional surface slope, the damping factor becomes

$$
\psi \approx \frac{\mathrm{e}^{\omega Z}-\mathrm{e}^{-\omega Z}}{\bar{\alpha}(\omega Z)^{2}}=\frac{\mathrm{I}}{\bar{\alpha} \Psi(\omega Z)} \quad \text { say. }
$$

For this same reason the average surface slope appears in Yosida's (1964) results for a viscous medium with no sliding while the individual parameters, $\eta, V, H$ do not. For nonlinear flow it is possible for $V_{\mathrm{s}}$ to be greater than $\rho g Z^{2} \bar{\alpha} / 4 \eta$ and hence $\psi<\mathrm{r} / \bar{\alpha} \Psi(\omega Z)$. However, in practice the damping factor is still generally large, but if necessary the full expression in Equation (4.32) may be used.

The function $\Psi(\omega Z)=(\omega Z)^{2} /\left(\mathrm{e}^{\omega Z}-\mathrm{e}^{-\omega Z}\right)$ for the frequency dependence of the damping factor is shown in Figure 3. This function has a maximum of $\Psi_{\mathrm{m}} \approx 0.552$ for

$$
\omega Z=2 \tanh \omega Z \quad \text { or } \quad \omega Z \approx 1.92
$$

and tends to zero as $\omega Z \rightarrow 0$ and as $\omega Z \rightarrow \infty$. Hence for surface slopes typically found on large ice masses $\left(\alpha \approx \mathrm{IO}^{-1}\right.$ to $\left.\mathrm{IO}^{-3}\right)$ the damping factor is large, i.e. $\psi \gg \mathrm{I}$. This justifies the approximation of Equation (4.33) and allows the shape of the surface to be expressed simply.

and

$$
\begin{aligned}
& \alpha_{\mathrm{S}} \approx \alpha_{2} \approx \frac{\beta_{1}}{\psi} \\
& \alpha_{\mathrm{I}}=\frac{\alpha_{2}}{2 \psi} \approx \frac{\beta_{1}}{2 \psi^{2}}
\end{aligned}
$$

where $\psi$ is defined by Equation (4.34). The phase lag between the surface and base is given by,

$$
\tan \delta=\frac{\alpha_{2}}{\alpha_{1}}=-\frac{1}{2 \psi}
$$

which shows that the surface is approximately $\frac{1}{2} \pi$ out of phase with the base. The signs in Equations (4.29), (4.30), (4.3 I) indicate that the steepest surface slope occurs over the highest point of the bedrock. 
From Equation $\left(4 \cdot 3^{8}\right)$ it is evident that the wavelength of minimum damping $\lambda_{\mathrm{m}}$ is given by,

$$
\frac{\lambda_{\mathrm{m}}}{Z} \approx \frac{2 \pi}{\mathrm{r} \cdot 9^{2}} \approx 3 \cdot 3
$$

i.e. $\lambda_{\mathrm{m}}$ is about three times the ice thickness. Figure 3 indicates that the maximum is fairly broad so that $\Psi$ is within $90 \%$ of $\Psi_{\mathrm{m}}$ for $\lambda / Z$ between 2.4 and 4.5 .

For a bedrock profile consisting of a distribution of many harmonics of different wavelengths and amplitudes, Equation (4.34) for $\psi$ may be regarded as a "transfer function" or "filter" by which the surface profile may be calculated.

Finally to examine the magnitude of the shear stress near the surface compare Equation (4.4) with Equation (4.13) to obtain,

$$
\left.\tau_{x z}\right)_{Z}=2 \eta V \omega^{2} h .
$$

Using Equation $\left(4 \cdot 3^{6}\right)$ this may be written,

$$
\left.\tau_{x z}\right)_{Z}=\frac{1}{2} \rho g \bar{\alpha} h(\omega Z)^{2} .
$$

Since for short waves $\alpha$ and $h \rightarrow 0$ exponentially as $(\omega Z) \rightarrow \infty$, this result for the effect of the undulations on the surface shear stress is quite compatible with the model. For both very long and very short waves $\left.\tau_{x z}\right)_{Z}$ becomes negligible and for $\lambda=2 \pi Z$,

$$
\left.\tau_{x z}\right)_{Z}=\frac{1}{2} \rho g \bar{\alpha} h
$$

as would be expected for the simplest approximation.

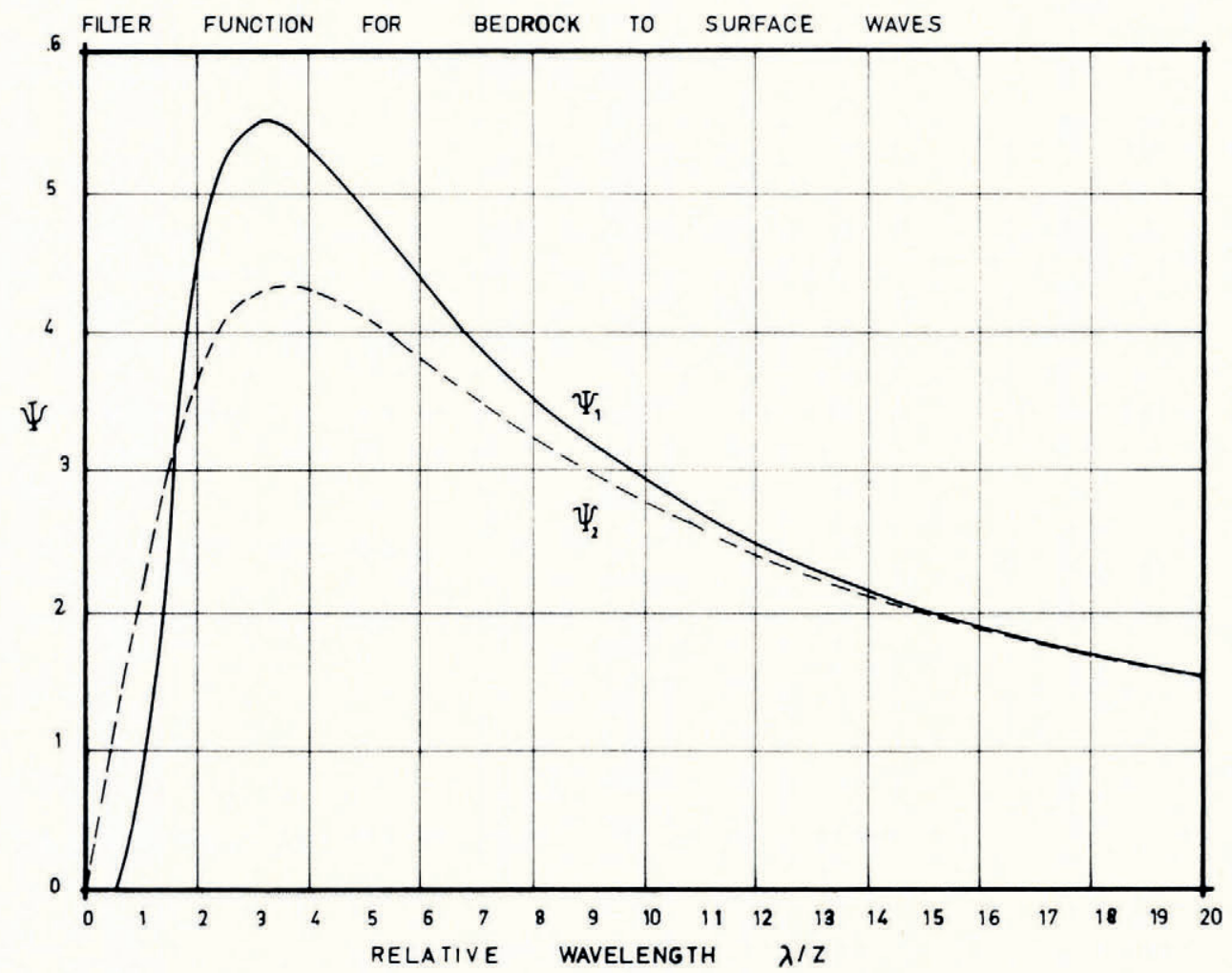

Fig. $3(a)$. The filter function $\Psi$, for the frequency-dependent part of the ratio of the amplitude of the surface and bedrock waves, is shown on linear co-ordinates against the ratio of the wavelength $\lambda$ to ice thickness $Z$. $\Psi_{1}(\omega Z)=(\omega Z)^{2} / 2$ sinh $\omega Z$ is from the present theory, $\Psi_{2}(\omega Z)=\omega Z / 2\left\{\mathrm{I}+\frac{1}{3}(\omega Z)^{2}\right\}$ is from Budd $(1969)$. 


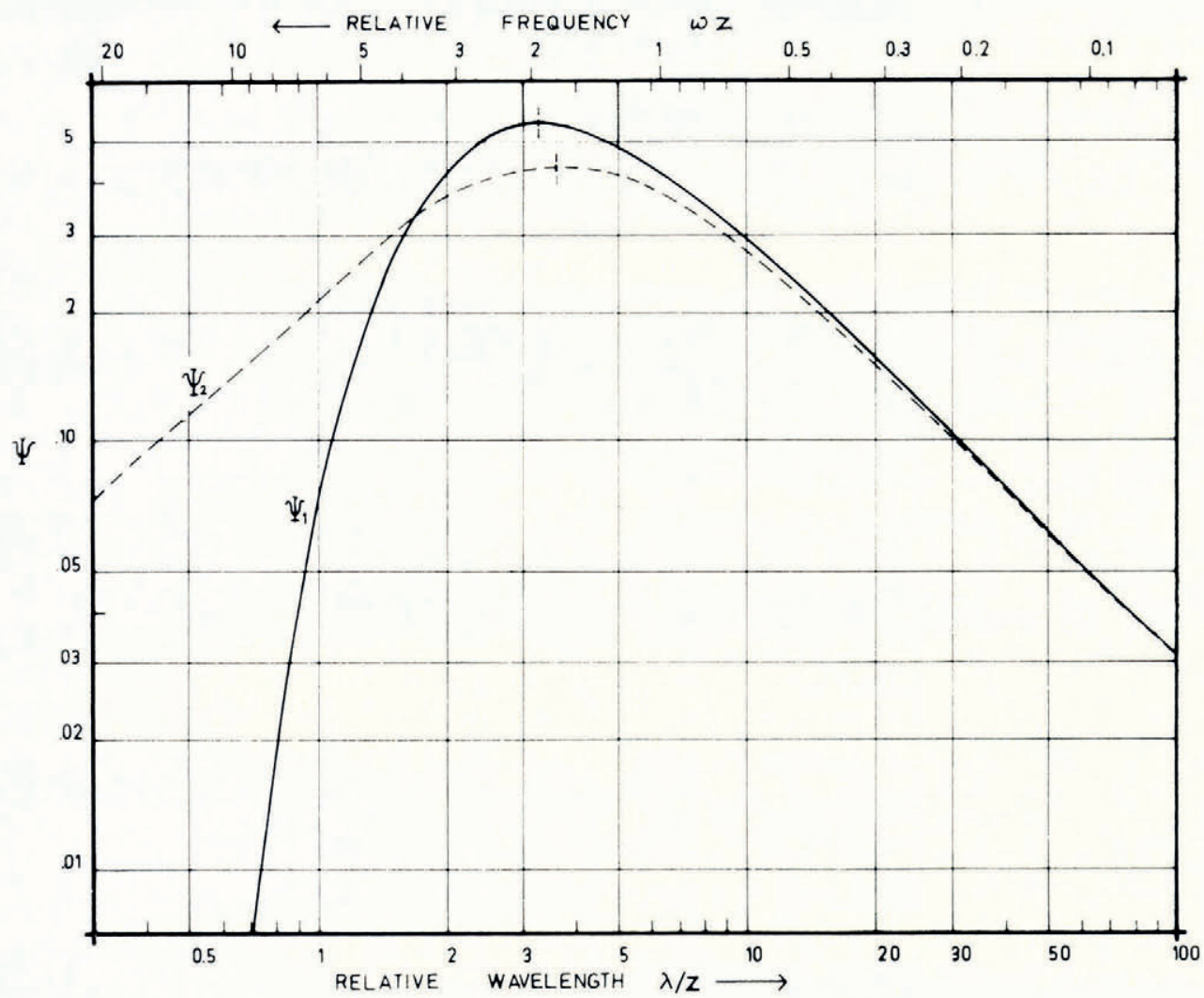

Fig. $3(b)$. The filter functions $\Psi_{1}$ and $\Psi_{2}$ versus $\lambda / Z$ on a $\log -\log$ scale.

\section{Applications}

\subsection{Wavelength of minimum damping}

The existence of a wavelength of minimum damping means that given a uniform distribution of bedrock variations we may expect the surface variations to have a distribution given by the transfer function,

$$
\alpha_{\mathrm{s}}=\frac{\beta_{1}}{\psi}=\frac{\beta_{14 \eta V}}{\rho g Z^{2}} \frac{(\omega Z)^{2}}{2 \sinh \omega Z}
$$

with a predominant wavelength about,

$$
\lambda_{\mathrm{m}} \approx 3.32
$$

The occurrence of surface waves of about this scale is well known on ice sheets, and this provides useful means of determining the flow properties of the ice (cf. section 5.2). Brockamp and Thyssen ([1968]) have also found them useful in determining the velocity of the ice.

The wavelength of minimum damping does not depend on the "viscosity" but only on the ice thickness, varying typically from $c$. I $\mathrm{km}$ or less near the edge to $c$. Io km inland on the large ice sheets. Beitzel (unpublished) confirmed the wavelength dependence of the damping factor for longer waves ( 5 to $5^{\circ} \mathrm{km}$ ) by spectral analyses of the surface and bedrock variations over a $400 \mathrm{~km}$ section of the "Queen Maud Land Traverse" in Antarctica $(Z \approx 2.7 \mathrm{~km})$. Carter (in preparation) has confirmed its validity for the Wilkes ice cap $(Z \approx 1 \mathrm{~km})$ extending the values to the very short waves $(\approx 0.4 \mathrm{~km})$, which clearly supported the exponential 
damping of Equation (4.34) rather than the earlier result, Equation (1.6). For long waves the two expressions do not differ greatly.

Similar analyses for the surface slopes of the Wilkes-Vostok (Antarctica) and the E.G.I.G. (Greenland) profiles have shown the systematic linear variation of the wavelength of maximum surface slope deviation with ice thickness. ${ }^{*}$

It is of interest whether such features are as prevalent on the surfaces of temperate glaciers. The variations in the surface slope measured by Paterson and Savage ( 1963 ) along the centre line of the Athabasca Glacier suggest that this may be so (cf. Budd, I969, fig. 6. I).

\subsection{The magnitude of the damping factor}

The variation in the magnitude of the damping factor with ice thickness, velocity and viscosity is the same for all relative wavelengths $(\lambda / Z)$. The magnitude of the damping factor for a particular wavelength provides a valuable means of determining the average value of the flow parameter through the ice, given the ice thickness and the velocity; viz. from Equation $(4 \cdot 34)$.

$$
\eta_{\theta}=\frac{\alpha_{\mathrm{s}} \rho g Z^{2} \sinh \omega Z}{2 \beta_{\mathrm{I}} V(\omega Z)^{2}}
$$

Values of the flow parameter calculated from this equation for the Wilkes ice cap have been found to agree with the corresponding values calculated in the same area from the direct measurements of the variations in surface slope and strain-rate over undulations (Budd, I969; L. Pfitzner†).

Carter has also shown that the variations in the damping factor from the summit of the Wilkes ice cap to the coast are in accord with the measured ice velocities and the calculated temperature-depth profiles. These temperature profiles have been found by Carter to give variations in dielectric absorbtion which matched the regional variations in the radar attenuations measured with the ice-thickness sounder.

\subsection{Relation between surface and bedrock topography}

Since the perturbation solutions for different frequencies are superimposable, it is possible to analyse the bedrock profile in a series of Fourier harmonics, and to apply Equation (4.34) for the damping factor as a transfer function to obtain the surface profile, provided the flow is sufficiently two-dimensional. This has been carried out with success by Carter $\ddagger$ for part of the Wilkes ice cap. Alternatively if the bedrock is broadly known (e.g. from seismic studies) it is possible to infer the short-wave bedrock variations from the surface variations. Obviously however, the latter can only be accurate for a limited range of wavelengths around that of minimum damping (c. $3 Z$ ), say $0.8 Z$ to $30 Z$, which nevertheless is the most important range for the ice motion, as is shown in the next section.

Further general relationships between the bedrock and the surface may be obtained by the spectral analyses described previously. The precise phase shift in the undulations for the different frequencies can be determined by the relative magnitudes of the co-spectra and quad-spectra of the surface and bedrock elevation deviations from a smooth trend. A further example of the correspondence between surface and bedrock deviations is given by Hochstein ([1967]) for west Antarctica for undulations of wavelength of about $30 \mathrm{~km}\left(\approx \mathrm{r}_{5} Z\right)$. Here again the phase shift of about $\frac{1}{2} \pi$ is indicated.

\footnotetext{
* Budd, W. F., and Carter, D. Analysis of the relation between the surface and bed-rock of ice caps. [In preparation.]

$\dagger$ Wilkes ice cap project 1966 . [To be published as an ANARE Scientific Report.]

$\ddagger$ Wilkes ice cap project 1967 , investigations by radar sounding. [To be published as an $A \mathcal{N} A R E$ Scientific Report.]
} 
5.4. Basal stress and glacier sliding

It is evident from the formula for shear stress (4.4) that at the base, using Equation (4.9),

$$
\left.\tau_{x z}\right)_{\mathrm{b}}=2 \eta V \omega \beta \cot \omega x,
$$

i.e. the maximum shear stress occurs at the tops of the waves and the minimum in the troughs.

Furthermore for waves of the same shape $\left(\beta_{1}\right.$ constant) the shear stress increases with frequency $\omega$. However, from Equation (4.4) it is also evident that the influence of the waves dies out exponentially $\left(\right.$ as $\left.\mathrm{e}^{-\omega z}\right)$ with distance into the ice such that one wavelength into the ice it has been reduced to $\mathrm{e}^{-2 \pi} \approx \mathrm{I} / 500$ of its value. This effect is even more enhanced for a high power-law flow or for cold ice caps where the "effective viscosity" is greatest near the base. Hence the small high-frequency waves have very little direct effect on the motion of the ice and only have an indirect effect in so far as the average bedrock stress over long distances determines the steady-state ice mass slope $\bar{\alpha}$, according to Equation (I.I). For small highfrequency variations $(\lambda \ll 3 Z)$ the term,

$$
T=\int_{0}^{z} \int_{0}^{z} \frac{\partial^{2} \tau_{x z}}{\partial x^{2}} \mathrm{~d} z \mathrm{~d} z
$$

is negligible. The energy dissipation due to the irregularities,

$$
E \propto \int\left\{\tau_{x z} \frac{\mathrm{d} u}{\mathrm{~d} z}+\left(\sigma_{x}-\sigma_{z}\right) \frac{\mathrm{d} u}{\mathrm{~d} x}\right\} \mathrm{d} z
$$

also has a maximum for wavelengths several times the ice thickness. Hence it is bedrock irregularities of these magnitudes which are most important in controlling the ice motion.

In contrast to the short-wave irregularities, which are less important, the bedrock undulations with wavelength several times the ice thickness can be readily determined by radar sounding and even gravity techniques.

The detailed study of these effects and their relevance to glacier sliding will be presented by the author in a separate paper.*

\subsection{How far can ice travel uphill (i.e. with the surface sloping downwards in the opposite direction to travel)}

The question of ice travelling uphill has been raised by Nye (1965, 1966) and Shumskiy $($ i 965, г $966[\mathrm{a}],[\mathrm{b}])$.

It appears from the surface profiles in Greenland (Mälzer, I964; Brockamp and Thyssen, [r968]) and Antarctica (Budd, r966, fig. o.5; Beitzel, unpublished) that ice can travel uphill for several kilometres.

The preceding analysis allows an analytical appraisal of the problem. Although a complete discussion will not be attempted here because of the assumption of a given ice thickness with small bedrock perturbations (cf. section 6.5) a criterion can be established for the conditions required for ice to travel uphill and for how far it can travel.

From Equations (4.I I) and (4.39) the surface slope may be written as,

$$
\alpha=\bar{\alpha}+\alpha_{\mathrm{s}} \cos \omega x \text {. }
$$

The condition for the flow to be uphill is that $\alpha$ becomes negative, i.e.

$$
\alpha_{\mathrm{s}} \cos \omega x>\bar{\alpha} \text {. }
$$

From Equation (5.I) this may be written

$$
\beta_{1} \cos \omega x / \psi>\bar{\alpha} .
$$

* Sliding and basal stress of ice masses. [Paper in preparation.] 
Taking $\cos \omega x=\mathrm{I}$ for the case in which the slope just becomes negative,

$$
\beta_{\mathrm{I}}>\bar{\alpha} \psi=\frac{\rho g Z^{2} \bar{\alpha}\left(\mathrm{e}^{\omega Z}-\mathrm{e}^{-\omega Z}\right)}{4 \eta V(\omega Z)^{2}} .
$$

It is apparent that the conditions for uphill flow are most advantageous near the wavelength of minimum damping $\lambda_{\mathrm{m}}$ for which, from Equation $\left(4 \cdot 3^{8}\right)$,

Since

$$
\begin{gathered}
\psi_{\mathrm{m}} \approx \frac{\rho g Z^{2} \bar{\alpha}}{4 \eta V} \\
\beta_{\mathrm{I}}=-\omega b_{\mathrm{I}}=-2 \pi b_{\mathrm{I}} / \lambda
\end{gathered}
$$

the criterion (5.10) may be written, for magnitudes,

Taking $\lambda$ near $\lambda_{m}$ say, e.g.,

$$
2 \pi b_{\mathrm{I}} / \lambda \approx \bar{\alpha} \psi_{\mathrm{m}} \text {. }
$$

this becomes,

$$
\lambda=\pi Z \text {, }
$$

$$
\frac{b_{1}}{Z} \approx \frac{1}{2} \bar{\alpha} \psi_{\mathrm{m}} \simeq \frac{\rho g Z^{2} \alpha}{4 \eta V}
$$

For a viscous medium with no slip,

$$
V=\frac{\rho g Z^{2}}{4 \eta}
$$

which implies the bedrock perturbation would have to be the order of the ice thickness. For the model treated here with base sliding or a "non-Newtonian basal boundary layer" the condition is equivalent to,

$$
\frac{b_{1}}{Z}=\frac{\eta_{2} Z}{\eta_{1} \Delta Z}
$$

where $\eta_{2}$ and $\eta_{1}$ are the "average viscosities" of the boundary layer and column respectively and $\Delta z$ and $z$ are their corresponding thicknesses. Hence the condition reduces to one in which the basal layer has a substantially lower effective viscosity than the upper layers. The higher temperatures and stresses near the base of cold ice caps contribute to this condition.

To consider some numerical values Beitzel (unpublished) gives a minimum damping of about 8 for a region where the ice surface has an average slope of about $0.25 \times 10^{-3}$. For this situation a negative surface slope occurs when, from Equation (5.15), $b_{1} / Z>\frac{1}{2} \times 2.5 \times 10^{-3} \times 8$, i.e. $b_{1}>Z / 100$ or for $Z=2.7 \mathrm{~km}, b_{1}>27 \mathrm{~m}$. For longer waves say $\lambda \approx{ }_{10} Z$ the corresponding values come to $b_{1}>Z / 20$, i.e. $b_{1}>135 \mathrm{~m}$. Finally the distance over which the ice will flow uphill is given by Equation (5.9) as,

or

$$
\begin{aligned}
& x=\frac{\lambda}{2 \pi} \cos ^{-1} \frac{\bar{\alpha} \psi}{\beta_{1}} \\
& x=\frac{\lambda}{2 \pi} \cos ^{-1} \frac{\bar{\alpha}}{\alpha_{\mathrm{s}}} .
\end{aligned}
$$

Hence for typical values of surface-slope variations, for a given average slope, it is seen that ice may travel uphill for several times its own thickness.

\subsection{Transient surface disturbances}

Steady-state surface variations do not exist without corresponding bedrock variations (provided they are not perpetuated by accumulation or ablation variations, cf. Black and Budd (1964), Nye (1959[a]).

The rate at which such surface disturbances diminish has been given by Jaeger ( 1962 , p. I 43) or Lamb (1932, p. 624) for a viscous semi-infinite medium as follows. 
For a harmonically disturbed surface with elevation variations given by,

$$
h=h_{\mathrm{o}} \cos \omega x
$$

so that the vertical stress variations at depth $z$ may be taken as

$$
\sigma_{z}=-\rho g h_{0} \mathrm{e}^{-\omega Z} \cos \omega x
$$

the vertical velocity follows from the stream function and may be written

$$
u=-\frac{\rho g h_{0}}{2 \eta \omega} \mathrm{e}^{-\omega Z} \cos \omega x .
$$

Hence the rate of lowering of the top of a crest is given by

or

$$
\begin{gathered}
\left.\frac{\mathrm{d} h}{\mathrm{~d} t}\right)_{\substack{x=0, z=0\\
}}=-\frac{\rho g h}{2 \eta \omega} \\
h=h_{0} \exp -\left(\frac{\rho g t}{2 \eta \omega}\right)=h_{0} \exp -\left(\frac{\rho g \lambda t}{4 \pi \eta}\right) .
\end{gathered}
$$

In contrast with the perturbations discussed by Nye (1959[b]), the perturbations dealt with here die out more rapidly for larger wavelengths and apply only to perturbations of wavelengths small compared with the ice thickness.

For long waves it is necessary to consider the case of a finite slab of thickness $Z$ say. The corresponding solution may be obtained from the preceding analysis as

or

$$
\frac{\mathrm{d} h}{\mathrm{~d} t}=\frac{\rho g h}{2 \eta \omega} \tanh \omega Z
$$

Since

$$
h=h_{0} \exp -\left(\frac{\rho g Z \tanh \omega Z}{2 \eta \omega Z}\right) t .
$$

$$
\frac{\tanh \omega Z}{\omega Z} \simeq I_{1} \quad \text { as } \quad \omega Z \rightarrow 0,
$$

the limiting value of the time constant $k$ for the rate of dissipation of long waves becomes

$$
k=2 \eta / \rho g Z \text {. }
$$

This limiting value, however, only applies for zero basal friction.

\section{Limitations and extensions}

\section{I. Constant viscosity or power law or plastic flow}

The present model which considers a linear relation between longitudinal stress and strainrate in the upper layers does not have a serious restriction on its generalization. The condition of constant viscosity for longitudinal stress and strain-rate was not introduced until Equation (4.6). Hence the form of the solution for stresses is retained whatever the flow law. Also since the boundary condition for the surface involves the integral throughout the ice thickness of $\dot{\epsilon}_{x}=-\dot{\epsilon}_{z}=\left(\sigma_{x}-\sigma_{z}\right) / 4 \eta$, it is the weighted average of the flow parameter through the ice thickness which is relevant. Since the weighting factor is $\mathrm{e}^{ \pm \omega Z}$, only the short waves are greatly affected by a non-constant $\eta$ along the vertical.

To study this effect, a further extension to the present model may be made by considering a vertical variation in the flow parameter resulting from a stress-strain relation of the form,

$$
\dot{\epsilon}_{x}=\frac{\tau^{n-1}}{B^{n}} \sigma_{\dot{x}}^{\prime}
$$

where $\tau$ is the octahedral shear stress at depth $z$. 
For small longitudinal stress deviations $\left(\sigma_{x}^{\prime}\right)$ the octahedral value is almost proportional to the vertical shear stress $\left(\tau_{x z}=\rho g \alpha z\right)$, which varies linearly with depth $z$. Hence in the case of an isothermal medium the average longitudinal strain-rate throughout the column may be calculated from terms of the form,

where $\zeta=(z-z)$.

$$
\int_{0}^{z} \zeta^{n-1} \mathrm{e}^{ \pm \omega \zeta} \mathrm{d} \zeta
$$

This procedure may be useful for temperate glaciers, but for cold ice caps the most important variable for the flow law is the temperature. For typical temperature-depth profiles the warmest layers are near the base, so that incorporating a temperature effect in the flow law enhances the effect of the stress as described above, i.e. the basal layers have the lowest effective viscosity. The net result of both these effects is that the small wavelength perturbations are damped out even more rapidly than exponentially, whereas the long waves, which depend more on the average flow parameters for the column, are not greatly affected.

This extension to vertical stratification can be most satisfactorily treated in particular cases where the temperature and velocity depth profiles are known.

\subsection{Two-dimensional flow (plane strain-rate)}

In the practical investigations of the predictions of this theory the problem arises that most of the bedrock variations have an appreciable three-dimensional character. In these cases part of the flow goes around the obstacles rather than over them. At right angles to the direction of flow we may expect the bedrock and surface elevation variations to be in phase. This has been demonstrated for the Wilkes ice cap by McLaren (I968, fig. 2 I). Hence for a line at an intermediate angle to the flow direction. We may expect the phase between surface and bedrock to be between $o$ and $\frac{1}{2} \pi$.

An ideal situation to test the results of the theory would be a region in which the ice thickness measurements indicate that the flow is largely two-dimensional. The effect of the three-dimensional motion on the longitudinal direction can be taken into account by the technique of Budd ( 1968 , section 4) when both transverse and longitudinal strain-rates are known.

\subsection{Effect of the sides of the ice mass}

The application of the present two-dimensional analysis to glaciers is complicated by the presence of the glacier sides, and the transverse gradients of stress and velocity. However, many results carry over to smooth symmetric glaciers of slowly varying cross-sectional shape. The generalizations required to apply this analysis to the flow along the centre line of such a glacier have been discussed by Budd (1969, section 5.2).

The effects of the irregularities in the sides of glaciers or ice shelves may be treated in a similar way to those due to irregularities in the bed here. It follows that such perturbations are also damped out exponentially with distance from the edge.

\subsection{Small average surface slope}

A more general form of Equation (I.I) for any slope has been derived by Budd (I970) from which the approximations for small slope may be seen. Primarily the restrictions are that,

$$
\sin \alpha \approx \tan \alpha \approx \alpha, \quad \cos \alpha \approx I \quad \text { and } \quad \alpha^{2} \ll \alpha .
$$

For surface slopes up to $20^{\circ}$ the errors may be small enough to make the present treatment useful.

In order to allow the boundary condition, $\left.\sigma_{z}\right)_{Z}=\rho g h$ to be used for appreciable slopes, a factor $\cos \alpha$ must be incorporated. For very steep glaciers a special treatment would be required. 


\subsection{Large perturbations}

The author believes that the restriction to small perturbations is not as serious as the restriction to two-dimensions. If the flow over a large obstacle is completely restricted to two-dimensions, one may expect the ice mass to increase in thickness sufficiently so as to be able to flow over the obstacle and still maintain a state of balance.

The present analysis uses a given ice thickness. It may be possible in future to use variational methods to determine the way in which the ice would flow down the slope over the obstacles determining its own thickness for a steady-state with the minimum dissipation of potential energy. With these theoretical considerations in mind the author would be glad to know if any field situations exist for steady state two-dimensional flow, in which the obstacle amplitude is large, say greater than one-third of the ice thickness. Such an example would provide a valuable means of studying the ice-flow mechanisms.

\section{ACKNowledgements}

The author is indebted to Dr J. F. Nye of the University of Bristol for drawing his attention to the important work of Yosida (I 964). Appreciation is extended to Dr P. A. Shumskiy of the Akademiya Nauk, Moscow for forwarding an early reprint of his 1967 publication. The author is grateful to Messrs J. E. Beitzel of the University of Wisconsin, and D. Carter of the Antarctic Division, Melbourne for making available the results of their analyses prior to publication. Dr U. Radok of the University of Melbourne kindly read the present manuscript and provided helpful suggestions on its presentation. Publication of this paper has the permission of the Director of the Antarctic Division, Department of Supply, Melbourne.

\section{MS. received II August 1969. Substantial proof revisions received 5 January 1970}

\section{REFERENCES}

Beitzel, J. E. Unpublished. The relationship of ice thicknesses and surface slopes in Queen Maud Land. [Paper read at International Symposium on Antarctic Glaciological Exploration, Hanover, N.H., U.S.A., 3-7 September 1968.]

Black, H. P., and Budd, W. F. 1964. Accumulation in the region of Wilkes, Wilkes Land, Antarctica. Fournal of Glaciology, Vol. 5, No. 37, p. 3-15.

Bourgoin, J.-P. I956. Quelques caractères analytiques de la surface et du socle de l'inlandsis groenlandais. Annales de Géophysique, Tom. 1 2, No. 1, p. 75-33.

Brockamp, B., and Thyssen, F. [1968.] Eine Anwendung der barographisch-topographischen Kurve zur Bestimmung der Bewegung von Inlandeisen. Polarforschung, Bd. 6, Jahrg. 37, Ht. I-2, 1967, p. 125-29.

Budd, W. F. 1966. Glaciological studies in the region of Wilkes, eastern Antarctica, 1961. ANARE Scientific Reports. Ser. A (IV). Glaciology. Publication No. 88.

Budd, W. F. 1968. The longitudinal velocity profile of large ice masses. Union de Géodésie et Géophysique Internationale. Association Internationale d'Hydrologie Scientifique. Assemblée générale de Berne, 25 sept.-7 oct. I967. [Commission de Neiges et Glaces.] Rapports et discussions, p. 58-77.

Budd, W. F. r969. The dynamics of ice masses. ANARE Scientific Reports. Ser. A(IV). Glaciology. Publication No. 108.

Budd, W. F. 1970. The longitudinal stress and strain-rate gradients in ice masses. Fournal of Glaciology, Vol. 9, No. 55 , p. $19^{-27}$.

Clough, J. W., and others. 1968. Ice-thickness investigations on SPQMLT III, by J. W. Clough, C. R. Bentley, and C. K. Poster. Antarctic Journal of the United States, Vol. 3, No. 4, p. 96-97.

Hochstein, M. P. [1 967.$]$ Morphologie der westantarktischen Eiskappe zwischen Mary-Byrd- [sic] und EdithRonne-Land. Polarforschung, Bd. 6, Jahrg. 35, Ht. I-2, 1965, p. 27-31.

Jaeger, J. C. 1962. Elasticity, fracture and flow; with engineering and geological applications. Second edition. London, Methuen. (Monographs on Physical Subjects.)

Lamb, H. 1932. Hydrodynamics. Sixth edition. Cambridge, University Press.

Lliboutry, L. I964-65. Traité de glaciologie. Paris, Masson et Cie. 2 vols.

McLaren, W. A. 1968. A study of the local ice cap near Wilkes, Antarctica. ANARE Scientific Reports. Ser. A(IV). Glaciology. Publication No. 103.

Mälzer, H. 1964. Das Nivellement über das grönländische Inlandeis der Internationalen Glaziologischen Grönland-Expedition 1959. Meddelelser om Gronland, Bd. I 73, Nr. 7. 
Mock, S. J. 1968. Snow accumulation studies on the Thule peninsula, Greenland. Fournal of Glaciology, Vol, 7, No. 49 , p. $59^{-76}$.

Nye, J. F. 1952. The mechanics of glacier flow. Fournal of Glaciology, Vol. 2, No. 12, p. 82-93.

Nye, J. F. 1958. A theory of wave formation in glaciers. (Cambridge Austerdalsbre Expedition.) Union Géodésique et Géophysique Internationale. Association Internationale d'Hydrologie Scientifique. Symposium de Chamonix, $16-24$ sept. $195^{8}$, p. 139-54.

Nye, J. F. 1959[a]. The deformation of a glacier below an ice fall. Journal of Glaciology, Vol. 3, No. 25, p. 386-408.

Nye, J. F. 1959[b]. The motion of ice sheets and glaciers. Journal of Glaciology, Vol. 3, No. 26, p. 493-507.

Nye, J. F. 1965. Theory of glacier variations; reply to Dr. Shumskiy's letter. Fournal of Glaciology, Vol. 5, No. 4o, p. 517-21. [Letter.]

Nye, J. F. 1966. Theory of glacier variations; reply to Dr. Shumskiy's letter. Journal of Glaciology, Vol. 6, No. 45, p. 465 . [Letter.]

Paterson, W. S. B. Unpublished. Observations on Athabasca Glacier and their relation to the theory of glacier flow. [Ph.D. thesis, University of British Columbia, Vancouver, B.C., Canada, 1962.]

Paterson, W. S. B., and Savage, J. C. 1963. Geometry and movement of the Athabasca Glacier. Fournal of Geophysical Research, Vol. 68, No. 15, p. 4513-20.

Robin, G. de Q. 1958. Glaciology. III. Seismic shootings and related investigations. Norwegian-BritishSwedish Antarctic Expedition, 1949-52. Scientific Results, Vol. 5.

Robin, G. de Q. 1967 . Surface topography of ice sheets. Nature, Vol. 215, No. 5105, p. 1029-32.

Robinson, E. S. 1966 . On the relationship of ice-surface topography to bed topography on the South Polar plateau. Journal of Glaciology, Vol. 6, No. 43, p. 43-54.

Shumskiy, P. A. 1965. Theory of glacier variations. Journal of Glaciology, Vol. 5, No. 40, p. 515-17. [Letter.]

Shumskiy, P. A. I966[a]. Theory of glacier variations; reply to Dr. Nye's letter. Journal of Glaciology, Vol. 6 , No. 44 , p. 3 19. [Letter.]

Shumskiy, P. A. $1966[\mathrm{~b}]$. Theory of glacier variations; reply to Dr. Nye's letter. Journal of Glaciology, Vol. 6, No. 45 , p. 465 . [Letter.]

Shumskiy, P. A. 1967 . The distribution of stress, velocity and temperature in glaciers. (In Oura, H., ed. Physics of snow and ice : international conference on low temperature science. ... 1966. ...Proceedings, Vol. I, Pt. I. [Sapporo], Institute of Low Temperature Science, Hokkaido University, p. $371-84$.

Sokolnikoff, I. S., and Specht, R. D. 1956. Mathematical theory of elasticity. Second edition, [by] I. S. Sokolnikoff. New York, McGraw-Hill.

Swithinbank, C. W. M. 1959. Glaciology. I. The morphology of the inland ice sheet and nunatak areas of western Dronning Maud Land.- The regime of the ice sheet of western Dronning Maud Land as shown by stake measurements. Norwegian-British-Swedish Antarctic Expedition, 1949-52. Scientific Results, Vol. 3, D, p. $97^{-11}$; ; Vol. 3 , E, p. 121-44.

Timoshenko, S. 1934. Theory of elasticity. New York, McGraw-Hill. (Engineering Societies Monographs.)

Yosida, Z. 1964 . Shamen sekisetsu no naibu ōryoku oyobi nensei ryūdō [Internal stress and viscous flow of snow covers on sloping ground surfaces]. Teion-kagaku: Low Temperature Science, Ser. A, [No.] 22, p. 83-127.

RÉsumÉ. Écoulement glaciaire sur des perturbations du socle rocheux. L'emploi de solutions périodiques simples et bien connues de l'équation de contrainte biharmonique à deux dimensions pour l'étude de l'écoulement sur des ondulations d'une masse de glace de faible pente de surface est examinée. Le modèle considéré est celui dans lequel la plus grande partie de l'effort de cisaillement (déformation ou glissement) prend place près de la base, la partie supérieure bougeant largement comme un bloc avec des vitesses de déformation longitudinales variant linéairement avec les déviations de la contrainte longitudinale. Pour les perturbations du socle rocheux d'une longueur d'onde donnée, la forme superficielle permanente consiste en vagues similaires mais hors de phase de $\frac{1}{2} \pi$, de sorte que la pente la plus forte arrive sur le plus haut sommet du socle rocheux; et l'amplitude est réduite par un facteur d'amortissement dépendant de la vitesse, viscosité, épaisseur de glace et longueur d'onde.

L'amortissement minimum a lieu pour $\lambda_{\mathrm{m}} \approx 3,3$ fois l'épaisseur de glace, tandis que des ondes plus longues ou plus courtes sont presque complètement amorties. La dissipation d'énergie et la résistance à l'écoulement glaciaire est aussi maximum pour une échelle d'ondulation de plusieurs fois l'épaisseur de glace, tandis que les effets des petites irrégularitiés basales meurent exponentiellement avec la distance dans la glace et ont seulement un effet aussi loin que la contrainte basale moyenne est liée à la pente moyenne de surface. En conséquence, une révision des théories de glissement actuel de glacier devient possible.

Des prédictions variées de la théorie ont été confirmées par l'analyse spectrale des profils de la surface et du socle rocheux des calottes glaciaires. 